\title{
ILCEA
}

Revue de l'Institut des langues et cultures

d'Europe, Amérique, Afrique, Asie et Australie

28 | 2017

Passages, ancrage dans la littérature de voyage

\section{La littérature de voyage et d'ascension : du passage de la relation de voyage à la conscience de la relation au monde}

Travel and Mountain literature: From Travel Reports to the Conscience of Man's Relationship with the World

\section{Françoise Besson}

\section{OpenEdition}

\section{Journals}

Édition électronique

URL : http://journals.openedition.org/ilcea/4133

DOI : 10.4000/ilcea.4133

ISSN : 2101-0609

\section{Éditeur}

UGA Éditions/Université Grenoble Alpes

Édition imprimée

ISBN : 978-2-84310-374-2

ISSN : 1639-6073

\section{Référence électronique}

Françoise Besson, «La littérature de voyage et d'ascension : du passage de la relation de voyage à la conscience de la relation au monde », ILCEA [En ligne], 28 | 2017, mis en ligne le 06 mars 2017, consulté le 30 avril 2019. URL : http://journals.openedition.org/ilcea/4133 ; DOI : 10.4000/ilcea.4133

Ce document a été généré automatiquement le 30 avril 2019.

(c) ILCEA 


\title{
La littérature de voyage et d'ascension : du passage de la relation de voyage à la conscience de la relation au monde
}

\author{
Travel and Mountain literature: From Travel Reports to the Conscience of Man's \\ Relationship with the World
}

Françoise Besson

1 Tout récit de voyage, en nous montrant un monde autre, éveille notre conscience de l'autre et du monde et nous guide dans notre «maison du monde » comme le dit Michel Serres: "La nouvelle vision du monde écologique part de l'idée que la terre est notre maison» (2016: 8), ce que dit aussi le chant navajo repris par N. Scott Momaday, dans La maison de l'aube, qui traduit le titre de son premier roman, House Made of Dawn.

2 Le choix de textes de fiction et de non fiction centrés sur le voyage et d'auteurs appartenant à des aires géographiques et à des époques différentes a pour but de montrer que ces différences ne séparent pas mais unifient la relation du voyage. Qu'il s'agisse des voyageurs anglais en visite dans les Pyrénées ou d'écrivains amérindiens, tel N. Scott Momaday dont l'œuvre est traversée par les voyages, de romanciers canadiens comme Rudy Wiebe dont le roman A Discovery of Strangers (1995) est une réécriture romanesque des journaux de la première expédition Franklin en quête du passage du Nord-Ouest, ou de Robert Hood, membre de cette première expédition, tous révèlent une conscience profonde du monde non humain et un regard aigu sur la relation de l'homme au monde. Liés par le voyage et le regard sur l'autre, qu'il soit humain ou non humain, des écrivains aussi différents que de simples touristes britanniques, des alpinistes, des romanciers amérindiens ou canadiens et des explorateurs anglais, suggèrent tous que la relation du voyage éclaire sur le lien de l'homme au monde, d'abord dans la vision de paysages vus dans des voyages de types multiples dont l'admiration esthétique va conduire à un sublime de type écologique. Dans tous ces récits, c'est l'observation du non humain, 
végétal ou animal, qui guide le lecteur à la fois vers la connaissance des écosystèmes et vers la conscience de leur fragilité et de la nécessité de leur préservation. Même la vision symbolique de l'animal dans la littérature amérindienne révèle le rapport entre la préservation des espèces et la survie de l'homme. La nomination des lieux et le rôle du toponyme révèlent deux conceptions de la relation de l'homme au monde, l'une qui parle d'appropriation et l'autre de conscience de la relation de l'homme à la terre. La représentation des paysages par des voyageurs et montagnards ${ }^{1}$ est-elle un moyen de préservation de ces espaces naturels? Et peut-on voir dans la littérature de voyage une tentative de sauvegarde de la planète? Les descriptions de la dégradation des paysages par la technologie ou l'exploitation guident le lecteur vers une prise de conscience et en cela peuvent le conduire à œuvrer pour la préservation des milieux naturels. C'est ce combat par la suggestion et la représentation que des textes aussi divers que des récits de voyages, d'ascensions, des romans et des essais liés au voyage peuvent suggérer.

\section{Rencontre de paysages}

3 Les rencontres de l'homme ou de la femme voyageurs ou alpinistes et de la montagne sont révélateurs du lien qui se tisse entre l'être humain et le monde non humain qu'il va rencontrer et toucher dans le cas de l'alpiniste. La montagne est le lieu du contact le plus étroit entre l'humain et le non humain. Elle est cet espace à la fois imaginé et touché qui révèle la force du non humain et la très illusoire puissance que s'arroge l'homme sur ce milieu. La montagne parle de l'autre. C'est donc dans cet ancrage profondément réel dans les sentiers des Pyrénées que les voyageurs anglais du XIX ${ }^{e}$ ont relaté leur expérience. Leur regard ethnologique qui savait percevoir toutes les différences en les rattachant à leur connaissance, car ils étudiaient aussi bien le costume et l'architecture des villages que les langues parlées tout au long de la chaine, s'accompagnait d'une conscience aiguë de la nature. Sans avoir encore conscience de l'orientation écologique de leurs récits - écologie avant l'heure puisque le mot n'est apparu sous la plume du zoologiste allemand Ernest Hackel qu'en 1874 - ils accordaient au monde animal et végétal une grande importance. Ils montraient tout ce milieu naturel qu'un alpiniste va nécessairement observer avec attention parce qu'il partage sa vie montagnarde avec lui. Pour si différente que soit l'œuvre romanesque et poétique d'un écrivain amérindien, on retrouve dans sa vision du monde cette conscience que le non humain parle au voyageur.

4 À travers l'œuvre du romancier et poète Kiowa N. Scott Momaday mais aussi d'auteurs amérindiens de nouvelles comme Joy Harjo, ainsi que dans l'étude des mythes amérindiens, se lit le rapport entre ces visions du monde et les déplacements d'ordre spirituel ou mythique (mais aussi réels) qui nous offrent un nouveau regard sur notre relation au monde. L'importance donnée par les Amérindiens et Premières Nations canadiennes au non humain - le voyage initial conduisant les hommes sur terre n'étant possible dans nombre de mythes que grâce à l'aide des animaux -, révèle que le voyage (qu'il soit touristique, voyage forcé dû à la guerre, pèlerinage ou voyage spirituel), et sa relation par le voyageur, ont une dimension écologique dans la mesure où ce regard voyageur n'exclut rien et ne place pas l'homme au centre de l'univers naturel, mais le présente comme une créature parmi d'autres qui ne peut exister sans l'aide des autres créatures.

5 Un récit de fiction peut éclairer sur la réalité d'un voyage d'exploration, comme le fait le roman de Rudy Wiebe (1995), A Discovery of Strangers, réécriture des journaux des 
explorateurs de la première expédition Franklin. L'écriture de Wiebe, dans ce passage de récits d'exploration à un récit de fiction, est centrée sur le regard porté sur l'autre ; en ouvrant son roman sur le point de vue animal, il révèle la conscience de la relation entre l'humain et le non humain et éclaire sur la dimension écologique du récit de voyage et de la littérature de fiction centrée sur le voyage. Aritha Van Herk (2014) évoque le lien qui existe entre l'admiration esthétique du lieu et une perception écologique: elle oppose l'âge de l'exploitation et de la domination au besoin de transcendance qu'offrent les paysages sauvages. L'admiration esthétique va conduire à ce qu'elle nomme «le sublime écologique » :

The ecological sublime insists on its own perpetuation; it tempts subscribers with the illusion of "the wonder, the inaccessibility of wild nature," and argues that "in an age of exploitation, commodification, and domination we need awe, envelopment, and transcendence" (Hitt, 1999: 620). While Burke's concept of the sublime expanded notions of the aesthetic, the drift of that lofty and much-abused capacity still inflects human approaches to wilderness. (Van Herk, $2014: 62$ )

6 L'idée de ce qui allait devenir l'écologie (l'étude de la science du milieu) apparaît notamment dans les récits de voyages et d'ascensions. Les voyageurs européens et américains découvrant les montagnes et les zones sauvages du monde admiraient leur beauté et en même temps étaient conscients des dangers qui commençaient déjà à les menacer. La mention de l'exploitation des forêts, des mines, de la construction des routes et des voies ferrées, de la mode de la chasse dite sportive, la cueillette massive d'espèces végétales destinées au commerce, rappelaient la transformation de la vie de la montagne et ces expériences personnelles suggéraient que le voyage pouvait prendre une dimension écologique.

Les récits de voyages ne sont pas simplement la relation d'une expérience individuelle décrivant un moment précis dans un lieu précis et évoquant le tourisme; ils ont aussi pour fonction de présenter à un lecteur la nature et la culture du pays ou de la région visités et guident vers la perception juste de l'autre, qu'il soit humain ou non.

\section{Le non humain pour guider l'homme}

Dans les récits de voyages ou de courses, l'observation de l'infiniment petit, la minuscule fleur ou l'insecte, conduit le voyageur ou le montagnard à avoir un rapport au monde plus humble. Il n'est pas le prédateur qui se sert de la nature, il est l'observateur qui la contemple et la comprend. La plupart du temps, les voyageurs donnent avec précision les noms des espèces végétales. La précision de ces listes botaniques et les descriptions des plantes participent aussi d'une démarche scientifique et rattachent ces textes du voyage aux ouvrages de ceux qui, revenus de longues expéditions, écrivent et dessinent à partir de leurs "voyages de découverte ». Mais la recherche botanique peut aussi avoir un rôle plus profond. Gaston Bachelard lie l'œil du botaniste à un retour à l'enfance :

L'« homme à la loupe » barre - bien simplement - le monde familier. Il est regard frais devant objet neuf. La loupe du botaniste, c'est l'enfance retrouvée. Elle redonne au botaniste le regard agrandissant de l'enfant. Avec elle, il rentre au jardin, dans le jardin [...]. Ainsi le minuscule, porte étroite s'il en est, ouvre un monde. Le détail d'une chose peut être signe d'un monde nouveau, d'un monde qui, comme tous les mondes, contient les attributs de la grandeur. La miniature est un des gîtes de la grandeur (Bachelard, $1992: 146$ ). 
Peut-être est-ce aussi un retour à l'enfance du monde, à un temps où les plus minuscules éléments de nature étaient inviolés. Et cette perception qu'a le voyageur ou le montagnard au regard de botaniste de la "grandeur » de la plus minuscule plante est peut-être le reflet de la conscience de la richesse du monde et de sa beauté, cette conscience qui laisse apparaître dans ces descriptions factuelles, une volonté de les préserver. Les voyageurs et montagnards anglo-saxons de passage dans les Pyrénées du $\mathrm{XIX}^{\mathrm{e}} \mathrm{au} \mathrm{XXI}^{\mathrm{e}}$ siècle laissent voir la flore pyrénéenne dans leurs récits. La liste de noms latins rappelle l'intérêt des voyageurs pour la botanique et notamment le goût de l'époque pour les fleurs alpines. Mais, s'opposant à la volonté de préservation marquée dans nombre de récits de voyages, les faits révélaient une toute autre attitude et les plantes, commandées par des amateurs, pouvaient parfois être arrachées par centaines comme le fut une espèce de narcisses de la vallée d'Arasas qu'un horticulteur anglais, Peter Barr, arracha de façon si définitive qu'il les fit disparaître dans cette zone. Les prélèvements étaient parfois aussi destinés à l'étude scientifique et commandés par des musées, mais dans ce cas, contrairement aux prélèvements sauvages à but commercial de Peter Barr, les prélèvements étaient constitués seulement d'échantillons, comme on le voit dans le roman canadien de Thomas Wharton, Icefields (1995), où l'un des personnages prélève dans les montagnes de ce qui allait devenir le parc national de Jasper au Canada des échantillons de fleurs destinées au musée d'Histoire naturelle de Londres. Et certains botanistes britanniques font un voyage pyrénéen exclusivement destiné à faire le relevé des plantes qui se rencontrent d'est en ouest, comme Georges Bentham.

L'observation de la montagne passe pour le voyageur botaniste par une observation de toutes les espèces de plantes qu'il traque inlassablement, Graal végétal qu'il poursuit dans une quête de voyage en voyage. John Muir, lors de ses voyages en Alaska, donne des listes de fleurs et de plantes pour montrer la richesse du lieu :

On the edge of some of the snow-banks I noticed cassiope. The thin, green, moss-like patches seen from camp are composed of a rich, shaggy growth of cassiope, white-flowered bryanthus, dwarf vaccinium with bright pink flowers, saxifrages, anemones, bluebells, gentians, small erigeron, pedicularis, dwarf willow and a few species of grasses. Of these, cassiope tetragona is far the most influential and beautiful. (Muir, $2002: 217$ )

11 La liste est clairement dominée par le cassiope, dont le nom revient trois fois dans ce court passage ; cette bruyère blanche arctique est montrée à la fois dans une vision esthétique (« beautiful »), à travers son origine mythique (son nom) et dans sa relation aux autres éléments (« influential»). Le botaniste se contente du nom de la plante comme si la nommer était d'une certaine façon se trouver soi-même. Et on peut penser à la quête inlassable de Théodore Monod qui, pendant quarante années, a parcouru le Sahara à la recherche d'une unique plante ou celle de John Muir qui a fait de même dans les montagnes de Californie à la recherche d'une orchidée rare. Bachelard a raison lorsqu'il dit que «la loupe du botaniste, c'est l'enfance retrouvée », et c'est aussi une manière de retrouver ce qu'il y a de plus profond en soi.

Pour comprendre la fonction des listes de plantes qui traversent nombre de récits de voyages, on peut partir de deux défenseurs de la nature. Jean-Jacques Rousseau et John Muir donnent peut-être la clé de la liste botanique. Jean-Jacques Rousseau, qui a écrit des Lettres élémentaires sur la botanique, dit dans la première lettre : " J'ai toujours cru qu'on pouvait être un très grand botaniste sans connaître une seule plante par son nom » (Rousseau, n. p.). C'est un paradoxe du philosophe qui pourtant s'intéressa à la botanique et à l'identification des plantes qu'il rencontrait. Il dit qu' « il faut voir avant de nommer » 
mais il parle bien de "nommer ». Et d'ailleurs, pour enseigner la botanique à une jeune élève, il utilise des termes techniques, se prenant tellement au jeu qu'il va écrire un Dictionnaire de botanique. Le fait que Rousseau, malgré son affirmation de départ, revienne vers la nomination, montre peut-être que nommer la plante est nécessaire pour la reconnaître, pour lui donner son identité. La plante existe dans la nature sans avoir besoin de nom. Mais la nomination est l'affirmation de la relation humaine à la plante (dès les textes bibliques, il est dit qu'Adam est chargé de donner un nom aux animaux : il leur donne une existence parce qu'il les nomme). De toutes les créatures vivantes, seul l'homme est capable de nommer. Les animaux ont un langage, ils savent communiquer, mais ils ne nomment pas. Le botaniste ou le voyageur qui se fait botaniste en insérant dans son récit des listes de plantes, révèle de façon originale la relation de l'homme à la nature. En nommant la plante, il affirme simplement son humanité, dans la joie de la rencontre avec l'autre non humain.

La liste reconstitue le paysage végétal et, en reproduisant la proximité des plantes sur la page, le voyageur donne une idée de l'écosystème. H.J. McKinder, dans sa Première ascension du Mount Kenya, insiste aussi sur la flore rencontrée durant l'ascension :

At one point we passed a rhinoceros skull, at another we heard an elephant trumpeting, and there were many evidences-freshly broken shrubs, fresh dung-which showed that elephants were very near. Yet everything seemed strangely familiar. There were stinging nettles with leaves only a little larger than those at home, equisetum, which clung to our clothes like the weed in our ditches, dead nettles, red, pink, and salmon-coloured pea blossoms, purple mallow, forget-me-not, thistle, clover, convolvulus, all no doubt, specifically different, yet producing a general impression similar to that of our English flora. For the rest, the flowers were the same as those in Meranga. A shrub with yellow blossom like laburnum, a pagoda-like spike with mauve circlets of blossom, white heliotrope, and large hanging bells of a pale yellow with a copper-coloured centre. Many of the trees had leaves like the laurel, the lilac, and the camelia. Evidently all Kikuyu was once clad with forest, and in Meranga, with the clearing of the forest, the wild flowers of the glades have overspread the whole country. Some Bates or Wallace ${ }^{2}$ should make this land his home for a couple of years before it is spoilt by civilization (McKinder, 1991 : 153).

Dans sa description, il mélange le familier et l'inconnu ("everything seemed strangely familiar » dit-il avec cet oxymore significatif), ce qui est typique du récit de voyage dans lequel les Européens comparent ce qu'ils découvrent et ce qu'ils connaissent dans leur pays d'origine. Il fait une liste des plantes rencontrées avant d'écrire: «Evidently all Kikuyu was once clad with forest, and in Meranga, with the clearing of the forest, the wild flowers of the glades have overspread the whole country ». Les descriptions précises que fait McKinder des plantes et des fleurs rencontrées opposent deux visions : il y a un fossé gigantesque entre l'esprit colonial passablement méprisant qui compare un Africain à un acteur dans le rôle de Mephistophélès dans un autre passage, et sa perception de la beauté pleine d'admiration lorsqu'il parle "du pays des Kikuyu autrefois vêtu de forêts " $^{3}$, où la métaphore du vêtement («clad ») suggère une communion entre l'homme et la nature. Le point de vue est double : la mention des naturalistes européens que le voyageur a envie de voir venir est dans la mouvance coloniale de celui qui ne voit que des Européens pour traduire la richesse d'un lieu; et en même temps il montre une conscience aiguë de la destruction progressive de la nature par la "civilisation», c'est-à-dire la présence européenne. La coupe des arbres et la déforestation étaient l'œuvre des colonisateurs européens et le remplacement des arbres par des fleurs sauvages en est la conséquence puisque ces nouvelles plantes colonisent l'espace autrefois boisé. Le terme anglais " overspread", s'il n'a pas avec la notion de colonisation la même proximité que le terme 
français scientifique indiquant la prolifération d'espèces végétales détruisant les espèces endémiques, suggère malgré tout l'étendue d'une emprise qui peut faire du non humain végétal l'image de l'humain. Ainsi la simple liste botanique est bien plus qu'une belle peinture colorée; c'est une métaphore de la colonisation humaine et en même temps un signe inquiétant de la transformation du paysage modifié par la déforestation. Si le monde végétal tient un rôle important dans ces textes, le monde animal permet de situer l'homme dans un écosystème.

Dans les récits de voyages britanniques, se lit la perception du monde animal par ces voyageurs du XIX ${ }^{e}$ siècle qui montraient simplement à leurs compatriotes les animaux de ces montagnes : ours, isards, loups, aigles. Dès 1825 , le récit de voyage de Thomas CliftonParis montre que ce voyageur britannique était choqué par la disparition inquiétante des ours des Pyrénées. Il part d'un simple toponyme, «the Step of the Bear " pour évoquer ce problème :

The Step of the Bear is some twenty feet across, so Bruin ${ }^{4}$ must have been a wonderful beast, a fit inhabitant of this colossal region, which might well be fancied the abode of giants of "mighty bone and bold empire" [...]. Throughout the wild mountains of the Pyrenees, this animal reigns supreme, although of late years, it has become scarce from the extermination war that is waged against it. (Clifton-Paris, $1843: 130$ ) force et la violence de l'image montrent que le voyageur avait conscience de la rapidité à laquelle l'espèce disparaissait, ce qui s'est avéré. Dans un roman publié à peu près à la même période par un romancier irlandais, Thomas Grattan, Caribert, the Bear Hunter (1825), une scène de chasse est présentée selon le point de vue du chien, qui est clairement du côté des animaux chassés et non de celui des chasseurs qu'il accompagne. Il s'agit là d'une autre façon, plus satirique, de critiquer cette forme de chasse sportive. Dans ses Méditations sur la chasse (2006), José Ortega y Grasset évoque la nécessité des limites imposées à la chasse sportive comme le feront plus tard des écrivains chasseurs américains tels que Jim Harrison, Thomas McGuane ou Rick Bass :

À mesure même que l'efficacité de l'arme s'est accrue, l'homme s'est imposé des limitations face à l'animal, de façon à le laisser à son jeu, et à ne pas déséquilibrer excessivement le chasseur et sa proie ; comme si dépasser certaines limites dans cette relation allait annihiler la nature profonde de la chasse, transformée alors en pure tuerie et destruction. (Ortega, $2006: 62)^{5}$

La conscience de l'autre non humain passe aussi par une vision claire de l'écosystème et Clifton-Paris donne d'ailleurs des informations sur la faune pyrénéenne :

Besides the bear, the wolf and izard inhabit these mountains. The former is the Lupus Lycaon, the black wolf or lobo of Spain: it is stronger in the limbs and shoulders than the common species-and is generally found in rocky and elevated ranges. They are exceedingly shy and ferocious, and formerly frequented in vast numbers the passes of the Pyrenees, where they have been seen bounding from bush to bush by the side of a string of mules, watching an opportunity to select a victim. (Clifton-Paris, $1843: 131$ ).

Le voyageur donne à son lecteur toutes les informations techniques concernant le loup : son nom scientifique latin, tout comme son nom en anglais et en espagnol, sa constitution, son caractère et son comportement, rien n'est passé sous silence. Là où Victor Brooke, chasseur et largement responsable de l'extinction des bouquetins dans les Pyrénées évoque son «sport », Clifton-Paris parle de raréfaction des espèces. Le point de vue du scientifique rejoint celui du poète Grattan qui, dans sa scène de chasse, montre la triste fin de l'animal tué par des humains qui sont ceux qui ont « un regard sauvage ». 

monde animal apparaît sous un angle à la fois symbolique et révélateur de la conscience d'une communion entre l'homme et l'animal : l'Indien centaure qui ne fait qu'un avec son cheval ou l'ours qui devient l'autoportrait de Momaday dans deux œuvres graphiques, les vols d'oies sauvages qui parlent du cycle de la vie et de la mort, la pieuvre sauvée par le poète qui le conduit à s'interroger sur la mémoire animale, les nombreux animaux qui jalonnent l'œuvre de Momaday, montrent le respect profond de l'Amérindien envers ces derniers. Il montre clairement le lien entre l'animal et l'homme lorsqu'il confie symboliquement au bison le soin d'œuvrer en faveur de la préservation de l'héritage culturel des peuples d'Amérique à travers sa fondation, Buffalo Trust; il dit dans «Vision Statement » : " the buffalo is more than an animal. It is the sun's shadow. Our lives are bound to it. If it lives, we live. If it dies, we die. It is our life and our living shield " ". L'animal est le signe de notre survie et il montre clairement que l'extinction d'une espèce animale est un pas de plus vers notre propre extinction.

Comme les textes de Momaday, certains textes canadiens révèlent la dimension écologique d'une vision de l'animal qui contient le respect dont font preuve les Premières Nations et en même temps, l'affirmation d'une conscience animale traduite, comme chez Grattan un siècle plus tôt, par le point de vue animal qui ouvre par exemple le roman de Rudy Wiebe, A Discovery of Strangers. En amont de ce roman, le journal de l'un des participants à l'expédition, prématurément disparu, Robert Hood, est révélateur. Dans ses peintures et dessins, Hood a su peindre l'histoire de la nature arctique et des populations vivant dans ces espaces que les colons voyaient comme un "pays nu ", selon les mots de Richardson, autre membre de l'expédition, cités par Rudy Wiebe dans Playing Dead (2003). Le texte de Wiebe montre comment le regard de l'Européen, qui voit un vide là où la vie est partout présente, s'oppose à celui des autochtones qui savent voir et écouter l'espace. Hood, en tant que naturaliste et artiste, a su voir toute la vie humaine et animale de l'Arctique ; même s'il était taraudé par la faim, malade, il ne voyait pas le monde autour de lui seulement comme une ressource qui devait le nourrir ; son regard a contribué à ouvrir une ère nouvelle dans la représentation des animaux puisque, au lieu de les isoler, il les représentait dans leur milieu naturel, dans cet écosystème qui ouvre la conscience du spectateur à la relation entre les animaux, les plantes, l'homme et le monde qui l'entoure. En ne séparant pas, Robert Hood montrait une vision écologique de la nature au sens étymologique du terme "écologie", science de l'environnement. Le récit et les dessins de ce jeune marin envoyé par l'Angleterre coloniale pour trouver un passage mythique et renforcer la puissance de l'Empire britannique et sa domination du monde, apparaissent comme le signe de ce passage de la colonisation à la conscience. Sa manière de peindre les peuples autochtones mais aussi le monde non humain guidait le lecteur de son journal vers une conscience de réciprocité et d'union entre l'humain et le non humain, un regard sur l'Autre qui explique notre relation au monde.

\section{De la colonisation à la conscience}

21 De l'Afrique à l'Arctique, des textes de voyage ou de fiction montrent qu'il y a le même désir dans l'esprit des voyageurs de remplir les blancs d'une carte pas encore dessinée. La toponymie est une marque importante de cette réappropriation des terres de l'autre. Les noms de lieux montrent comment le nom reflète un pouvoir et exprime une réappropriation de la terre. Les noms canadiens liés à la famille royale anglaise (Alberta, 
Victoria Falls, Prince Edward's Island...) sont les éléments visibles de cette colonisation traduite sur les cartes. Ils remplaçaient les noms indiens qui, eux, parlaient simplement du paysage et de la nature. Les récits de ces voyages d'exploration apparaissent donc souvent comme la matérialisation écrite de cet esprit colonial sur lequel on posait le masque idéalisé de la connaissance ou du rêve exotique :

During the period [1720-1914] [...], travel writing became increasingly identified with the interests and preoccupations of those in European societies who wished to bring the nonEuropean world into a position where it could be influenced, exploited or, in some cases, directly controlled. In the case of Britain, the identification was particularly close. (Bridges, $2002: 53)$

La toponymie est l'image visible d'une langue dans le paysage, qui parle de la relation d'un peuple au monde. Le remplacement par les colons des noms de lieux indigènes par des noms européens était signe de leur volonté de s'emparer de nouveaux territoires en se les appropriant déjà par la langue. L'acte de renomination est à la fois un acte de désappropriation et d'appropriation. Ils introduisirent la religion chrétienne dans la topographie (Los Angeles, San Francisco) et projetèrent le vieux continent sur le nouveau (New York, New Brunswick, Nova Scotia), alors que les populations autochtones montraient dans les noms une relation directe à l'environnement. Dans son journal, Robert Hood évoque divers toponymes :

The Woody Lake is 13 miles in length, and a Small glassy channel at its northwestern extremity, leads to the Frog Portage, the source of the waters descending by Beaver Lake to the Saskashawan. The distance to the Missinnippi or Churchill River is only 380 yards, and as its course crosses the height nearly at right angles to the direction of the Grande Riviere, it would be superfluous to compute the elevation at this place. (Hood in Stuart Houston, 1994 : 107-108, c'est l'auteur de l'article qui souligne).

On note l'alternance entre noms européens et noms amérindiens, comme le Saskatchewan (qui vient d'un mot cri signifiant "rivière rapide») et le Missinnippi. Parler de l'eau, c'est parler de voyage et de colonisation. Mais si les noms de lieux son nombreux dans tous les récits de voyage, leur sens n'est pas toujours donné. Dans le récit de McKinder (1990), First Ascent of Mount Kenya, les toponymes foisonnent mais jamais il ne donne leur sens, pas plus que Henry Russell (1864) dans son voyage à travers l'Asie et l'Océanie, ni William Moorcroft, dans son récit, Travels in the Himalayan Provinces of Hindustan and the Punjab (1841). Pour eux, seule importe la dimension exotique du nom. Lorsque le voyageur fait l'effort de chercher le sens d'un toponyme, cela révèle une autre vision du monde puisqu'il veut pénétrer dans le monde de l'Autre et non plus seulement rester à la surface. Il cherche à voir l'Autre de l'intérieur à travers le nom qu'il a donné au lieu. La traduction des toponymes ou son absence est plus importante qu'il n'y parait car elle révèle le fossé qui existe entre le simple colonisateur et le voyageur qui cherche à comprendre l'Autre. Rudy Wiebe (1995) résume l'idée dans A Discovery of Strangers; les explorateurs voyagent sur une rivière qu'ils nomment Coppermine River. Mais le texte dit que le vrai nom donné à la rivière par les autochtones était Copperwoman River, du nom d'un personnage mythique. Les explorateurs, parce qu'ils n'ont pas compris le lien entre les hommes et le lieu, ont fait du nom un signe d'exploitation matérielle et ont effacé le mythe. Ceci montre le rôle du toponyme dans la conception qu'a l'homme de sa relation à la terre. Là où les autochtones voient le lien avec la mémoire collective, les colons y voient un lieu d'exploitation des ressources. Pourtant parfois, le voyageur peut comprendre le lien entre le lieu et le mythe et dans le nom donné, montrer le respect qu'il a pour l'Autre ; ainsi McKinder écrit : 
The Masai have a legend that their race originally came down on to the plains from Kenya, where dwelt their divine ancestor. I have therefore felt it appropriate to apply to the highest summit of the mountain the name of their hero chief, Batian, and to the second summit, only a little lower, the name of his son, Nelion. It will be remembered that we carried Lenana's knobkerry for a safeguard in Laikipia. (McKinder, 1990 : 192)

\section{action en tant que voyageur et alpiniste. Et le choix qu'il fait dans la nomination de deux sommets révèle son respect pour la mémoire mythique. N. Scott Momaday évoque le rôle profond du toponyme dans les cultures amérindiennes : \\ Where language touches the earth, there is the holy, there is the sacred. In our deepest intelligence we know this: that names and beings are indivisible. [...] If we are speaking of place, which is (or ought to be) a fundamental concept in our life, the particularity is critical. We know that we are (and where we are) with reference to the things about us, the points of reference in both our immediate and infinite worlds, the places and points in which we are born, grow old and die. There is in this simple cartology the idea of odyssey. And in odyssey there is story. (Momaday, 1997 : 124).}

Dans cette dimension philosophique et même sacrée du toponyme, Momaday montre la philosophie amérindienne du lieu et du nom et parle ainsi de la relation de l'homme au lieu et à la nature comme une relation de respect. Une anecdote est révélatrice. Il parle d'un jour où il prit dans sa voiture un jeune auto-stoppeur navajo et il écrit que, tandis qu'ils parlaient, « une chose remarquable se produisit» (1997: 125). L'écrivain avait appris quelques mots de navajo et, en navajo, il posa cent fois au jeune homme la question qui signifie «quel est le nom de ce lieu? » Et chaque fois, le garçon donnait le nom et Momaday en conclut ceci : «He was eminently familiar with the places that defined him. [...] Not only did he know them [...] but he knew their names » (1997: 126). Le nom du lieu est perçu comme signe de la familiarité de l'homme avec ce lieu. Tous ces récits littéraires sont autant de témoignages mais ils sont plus que cela dans la mesure où la représentation qui y est donnée des espaces traversés et des peuples rencontrés apparaît comme une manière de préserver la nature peinte par les mots.

\section{La préservation de la nature dans sa représentation?}

Représenter un paysage, c'est peut-être déjà une manière de le préserver, en montrant sa beauté avant que les changements ne le transforment. Les récits d'ascension révèlent une conscience éthique peut-être en raison d'une relation charnelle à la montagne. Barry Lopez évoque à propos de l'Arctique la relation charnelle qui existe avec la nature à travers la conscience de sa beauté absolue :

I had a clarity of mind that made the map in my lap seem both wondrous and strange in its approximations. I looked west into Mokka Fiord, to a chain of lakes between two whitish gypsum domes. Beyond was the patterned ground of the mesic tundra. The browns and blacks and whites were so rich I could feel them. The beauty here is a beauty you feel in your flesh. You feel it physically and that is why it is sometimes terrifying to approach. Other beauty takes only the heart, or the mind. (Lopez, 2001 : 404)

À travers la musique de la langue et la répétition d'un même son - l'allitération en [f] - il réunit la chair et les sentiments. Le paysage est une philosophie visuelle de la perception de l'altérité et de la nature et les montagnards ressentent plus que tout autre ce lien. La construction des routes dans les zones de montagne les inquiète souvent et leur peinture des paysages devient une manière d'œuvrer à leur préservation. Ainsi Kev Reynolds (2013), voyageur et montagnard anglais contemporain et auteur de plus de quarante 
guides et livres de voyages sur les montagnes du monde, parle d'une zone pyrénéenne lorsqu'il la vit pour la première fois, véritable jardin d'Eden couvert de fleurs de toutes parts. Lorsqu'il y revient des années plus tard, il décrit la transformation du paysage et cite un autre voyageur, le Gallois Harold Spender :

In 1897 Harold Spender came down the valley of the Esera from its source among the glaciers. In his account of the journey he mentioned this track: 'We passed the baths of Venasque... and a little below came across some Spanish workmen employed on a road in a desultory fashion. Whether that road will ever be finished is a matter that must rest on the knees of the gods.'

Now, as we came to the Baños de Benasque-Spender's baths of Venasque-I saw that the gods had made their decision. Below, on the broad river plain, a contractor's vehicle belched clouds of diesel smoke.

Dusk was drawing on by the time we turned the bend into the upper sanctuary, and we were still on the bulldozed track that has not been there 18 months before. It led deeper into the valley with an urgency I feared. A concrete ford had been created through the river, and where vehicles had used it their skidding tyres had ripped the vegetation on both banks. A once-sacred meadowland was scarred with dry mud and the imprints of wheels, not animals. Dwarf rhododendrons had been desecrated, and rainbow swirls of oil coloured puddles in the track.

A sense of foreboding hung over me, and with every step I slipped deeper into a pool of dejection.

Fifty metres from the site of the idyllic terrace on which Keith and I had camped, the rough track finally came to a halt. Three cars were parked there; two Spanish, one French. Cardboard boxes lay strewn among the shrubbery, rotting after a shower of rain. Wine bottles had been smashed against a rock. Toilet paper fluttered from the branches of a pine tree, and tin cans were rusting in the stream.

'Urban motorised man,' wrote Fernando Barrientos Fernandez, 'has no responsible conservationist regard for nature.' [...]

'Where,' I wondered, 'will the izard go to drink now?' (Reynolds : 2013, 21-22)

Plusieurs montagnards à différentes époques, forment une chaîne qui montre que destruction et pollution remplacent l'Eden de la première rencontre. Les couleurs des fleurs ont fait place aux teintes artificielles des déchets et au faux arc-en-ciel qui pollue l'eau de la montagne. La peinture paysagée devint un cri de révolte. D'un paysage sacré (" once-sacred meadowland») on passe à un paysage couvert de cicatrices («scarred»); le quasi anagramme résume la situation et le message : l'homme moderne ne voit plus la nature comme sacrée mais il la blesse irrémédiablement. La force des mots qui montrent la «beauté de la montagne » «désacralisée » par la « laideur des intrusions » pousse le lecteur à se révolter à son tour, à prendre conscience de la destruction de certaines régions montagneuses par la construction de routes destinés à amener toujours plus de touristes. L'interrogation finale sur l'isard qui ne trouvera plus de point d'eau pour s'abreuver montre que la vie de la montagne est un tout et que la pollution du lieu menace la vie dans son ensemble.

Les montagnards, qui vivent en communion avec la montagne, ont cette conscience éthique évoquée par Laurence Buell (1996) ${ }^{7}$ car ils sont en contact direct avec la nature. Si l'on ne peut pas considérer le récit de voyage comme littérature environnementale, on peut noter que partout s'y manifeste cette conscience de la nature et cette attention au non humain dont parle le critique. S.E. S. Allen, dans le récit qu'il fait d'une ascension dans les Rocheuses canadiennes en 1896, trace une carte géographique et géologique des lieux sur laquelle va se projeter sa perception personnelle et poétique :

The western, southern, and eastern ridges of Mount Temple meet in a summit of broken rock corniced to the N. Our camp was visible, a dash of white by the river, 4,800 ft below us. The 
Sentinel and the Cathedral looked insignificant beneath us, and the Wastach and Wenkchemna Valleys with their peaks and glaciers lay mapped out for our inspection. When we looked down upon the beautiful Wastach Valley, shining with its green meadows, its darker forests, its sapphire glaciers, its graceful peaks and silver stream, we seemed translated to some fair scene in the Italian Tyrol. The other valley was all ice and rock and snow, swept with avalanches from and guarded by the dark walls of the stately crest, and seemed like some frowning recess of the Oberland. (Allen, $1896: 223$ )

30 Ailleurs, il compare les glaciers à « un temple dionysiaque » qui se détache sur « un ciel grec ». À travers l'intégration visionnaire d'un paysage (les Alpes) à un autre (les Rocheuses), et l'insertion métaphorique d'un théâtre dionysiaque et d'un ciel de Grèce, le montagnard fait entrer son expérience individuelle d'alpiniste dans l'histoire humaine par la référence mythique et réelle au berceau des civilisations qu'est la Grèce. On retrouve cela avec Henry Russell (1888) qui voit des temples, des obélisques, des monastères bouddhistes, des idoles et des minarets dans les montagnes pyrénéennes, inscrivant ainsi ces montagnes à travers sa perception des lieux dans l'histoire religieuse et spirituelle du monde.

31 Le récit de voyage comme le récit d'ascension élargit toujours le local et le particulier au reste du monde: des projections d'espaces lointains sont courantes chez les voyageurs comme chez les montagnards. Henry Russell dans ses Souvenirs d'un montagnard (1888), regardant par exemple le paysage à partir d'un sommet des Pyrénées, a l'impression de voir la Mongolie et les montagnes de l'Altaï. H.J. MacKinder, dans le récit de son ascension du mont Kenya, évoque le Krakatoa, ce volcan du Pacifique situé entre Sumatra et Java. Le mélange de descriptions picturales et de projections d'espaces lointains sur les montagnes parcourues suggère que chaque lieu est intégré pour le voyageur à un grand tout, ce qui rejoint la conception amérindienne de l'univers fait de cercles concentriques.

Des routes et des sentiers pyrénéens parcourus par des Britanniques jusqu'au regard amérindien et aux grands espaces canadiens, la littérature parle d'une observation du monde non humain qui guide vers la relation entre ce monde et l'homme. Cette relation guide le lecteur vers une prise de conscience à la fois de la beauté et de la fragilité de ce monde non humain qu'il est invité tacitement à protéger. Si l'on accepte le passage de la lecture à la conscience, on peut voir dans les textes une arme pour sauver la planète et ceux qui l'habitent. Les dictateurs l'ont bien compris, qui assassinent et exécutent les poètes. Ken Saro Wiwa et ses huit compagnons ont été exécutés parce qu'ils se battaient, notamment par leurs textes, contre les dégâts faits par une compagnie pétrolière sur le territoire Ogoni au Nigéria. La conscience est acte car elle conduit à l'acte, comme chez le prix Nobel Wangari Maathai qui, en reboisant son pays avec les femmes kenyanes, a donné réalité au conte de Jean Giono, L'homme qui plantait des arbres. Passage de l'imaginaire d'un écrivain au monde réel d'un autre, l'acte de plantation mis en œuvre par des femmes est signe que tout devient possible dès lors que le regard sur le monde éclaire notre relation à "notre maison du monde ". C'est le passage de ce cheminement intérieur à la suggestion faite au monde, du lien existant entre nous et les autres mais aussi entre nous et le non humain qui nous fait vivre et nous apprend la vie si nous savons regarder, qui transforme l'acte de lecture en une action ou un combat pacifique. Est-ce une utopie ou une pensée naïve que de penser que la littérature a le pouvoir de préserver la planète et tous ses habitants? Ken Saro Wiwa a été exécuté mais sa voix demeure dans ses textes :

I have devoted my intellectual and material resources, my very life, to a cause in which I have total belief and from which I cannot be blackmailed or intimidated. I have no doubt at 
all about the ultimate success of my cause, no matter the trials and tribulations which I and those who believe with me may encounter on our journey. Nor imprisonment nor death can stop our ultimate victory ${ }^{8}$. (1995)

\section{BIBLIOGRAPHIE}

ALLEN Samuel. E. Stokes (1896), « Mountaineering in the Canadian Rockies », The Alpine Journal, XVIII(134), 223.

BACHELARD Gaston (1992), La poétique de l'espace [1957], Paris : Quadrige/Presses Universitaires de France.

BenthAm George (1826), Catalogue des plantes indigènes des Pyrénées et du Bas-Languedoc avec des notes et observations sur les espèces nouvelles ou peu connues ; précédé d'une notice sur un voyage botanique fait dans les Pyrénées pendant l'été de 1825, Paris : Madame Huzard.

BESSON Françoise (2000), Le Paysage pyrénéen dans la littérature de voyage et l'iconographie britanniques du dix-neuvième siècle, Paris : L'Harmattan.

BESSON Françoise (2010), Mountains Figured and Disfigured in the English-Speaking World, Newcastle upon Tyne : Cambridge Scholars Publishing.

BESSON Françoise, OMHOVERE Claire \& VentuRA Héliane [dir.] (2014), The Memory of Nature in Aboriginal, Canadian and American Contexts, Newcastle Upon Tyne : Cambridge Scholars Publishing. BRIDGES Roy (2002), « Exploration and Travel Outside Europe (1720-1914)», P. Hulme \& T. Youngs, The Cambridge Companion to Travel Writing, Cambridge : Cambridge University Press.

BuELL Lawrence (1996), The Environmental Imagination: Thoreau, Nature Writing and the Formation of American Culture, Cambridge : Harvard University Press.

CLIFTON-PARIS Thomas (1843), Letters from the Pyrenees, Londres : John Murray.

Duloum Joseph (1970), Les Anglais dans les Pyrénées et les débuts du tourisme pyrénéen (1739-1896), Pau : Les amis du musée pyrénéen.

GRATTAN Thomas (1825), "Caribert, The Bear Hunter ", High-Ways and By-Ways or Tales of the Roadside picked up in the French Provinces by a Walking Gentleman, Londres : G. and W. B. Whittaker (deuxième série).

HITT Christopher (1999), « Toward an Ecological Sublime », New Literary History, 30(3) : 603-623. Houston C. Stuart [dir.] (1994), To the Arctic by Canoe 1819-1821, the Journal and Paintings of Robert Hood, Midshipman with Franklin [1974], Montréal et Kingston, Londres, Buffalo : McGill-Queen's University Press.

Hulme Peter \& Youngs Tim (2002), The Cambridge Companion to Travel Writing, Cambridge :

Cambridge University Press.

LOPEZ Barry (2001), Arctic Dreams. Imagination and Desire in a Northern Landscape [1986], New York : Vintage Books.

Mackinder (Sir) Halford John (1900), « The Ascent of Mount Kenya », The Alpine Journal, XX(148). 
MACKINDER (Sir) Halford John (1991), The First Ascent of Mount Kenya [1990], Athènes : Ohio University Press.

Momaday N. Scott (1968), House Made of Dawn, New York : Harper and Row.

Momaday N. Scott (1997), The Man Made of Words, New York : St Martin's Griffith.

MOORCROFT William (1841), Travels to the Himalayan Provinces of Hindustan and the Punjab-In Ladakh and Kashmir in Peshawar, Kabul, Kunduz and Bokhara, Londres : John Murray.

MUIR John (2002), Travels in Alaska [1915], New York : The Modern Library.

OMHOVÈre Claire [dir.] (2014), L’Art du paysage, Paris : Michel Houdiart.

ORTEGA Y GASSET José (2006), Méditations sur la chasse [1942], Québec : Septentrion.

PRATT Mary Louise (1992), Imperial Eyes. Travel Writing and Transculturation, Londres : Routledge.

REYNOLDS Kev (2013), A Walk in the Clouds. Fifty Years Among the Mountains, Cumbria : Cicerone.

Rolland NABUCo Céline (2016), Figures, gestes et cibles du sportsman. La chasse dans les textes de Jim Harrison et Thomas McGuane (thèse de doctorat), Université de Toulouse.

RouSSEAU Jean-Jacques (2014), Lettres sur la botanique, Paris : Ink Book Edition.

RuSSELL Henry (1888), Souvenirs d'un montagnard (1858-1888), Pau : imprimerie Vignancour.

RuSSELl Henry (1864), Seize mille lieues à travers l'Asie et l'Océanie (2 vol.), Paris : Librairie Hachette et Cie.

SARO-WiWA Ken (1995), « Ken Saro-Wiwa's final address to the military-appointed tribunal », Earth Island Journal, 11(1), 25

SERRES Michel (2016), Darwin, Bonaparte et le Samaritain. Une philosophie de l'histoire, Paris : Le Pommier.

SPENDER Harold \& LleLLyn-SMITH (1898), Through the High Pyrenees, Londres : Sampson, Low, Marton and Company.

VAN HERK Aritha (2014), « Curtailed by the Sublime: Canada, Nature and the Contingencies of Beauty», F. Besson, C. Omhovere \& H. Ventura (dir.), The Memory of Nature in Aboriginal, Canadian and American Contexts, Newcastle Upon Tyne : Cambridge Scholars Publishing.

WIEBE Rudy (1995), A Discovery of Strangers, Toronto : Vintage Books.

WIEBE Rudy (2003), Playing Dead. A Contemplation Concerning the Arctic [1989], Edmonton : Newest Press.

WHARTON Thomas (1995), Icefields, Edmonton : NeWest Publisher.

\section{ANNEXES}

Françoise Besson est professeur de littératures de langue anglaise à l'Université Toulouse - Jean Jaurès. Sa recherche porte sur la relation entre paysage et écriture et entre littérature et écologie dans la littérature britannique, amérindienne et canadienne et en particulier dans la littérature de voyage et la littérature de la nature. Elle a publié ou dirigé et co-dirigé plusieurs ouvrages sur la montagne et la mémoire de la nature, notamment Le Paysage pyrénéen dans la littérature de voyage et l'iconographie britanniques au 
dix-neuvième siècle (2000), Mountains Figured and Disfigured in the English-Speaking World (2010) et, avec Claire Omhovère et Héliane Ventura, The Memory of Nature in Aboriginal, Canadian and American Contexts (2014). Elle est directrice éditoriale de la revue Caliban et présidente de la SELVA. Elle est aussi l'auteur de plusieurs recueils de poèmes, contes et nouvelles et co-auteur avec Madeleine Besson de plusieurs ouvrages d'histoire régionale.

\section{NOTES}

1. Si le terme de montagnard a été généralement choisi plutôt que celui d'alpiniste, c'est parce qu'il définit autrement la relation de l'homme à la montagne. L'emploi d'un terme plutôt que l'autre suscite des débats. L'alpiniste pratique un sport. Le montagnard, au départ, est l'habitant des montagnes. Mais nombre d'alpinistes ont choisi de se définir comme montagnards car dans ce terme, ils voient une relation plus profonde avec la montagne. Gaston Rébuffat écrivait «Avant la Verte, on est alpiniste, à la Verte, on est montagnard!» (<http://www.chamonixguides.com/Blog_article/246/article/

_avant_la_verte_on_est_alpiniste_a_la_verte_on_devient_montagnard_gaston_rebuffat/121-leblog.htm>, consulté le 22 octobre 2016). On peut aussi penser à Henry Russell (1888) qui a choisi d'intituler l'histoire de ses ascensions relatant sa communion avec la montagne, Souvenirs d'un montagnard.

2. H. W. Bates était un naturaliste auteur de A Naturalist on the River Amazon (1863) et A. R. Wallace est l'auteur de Travels on the Amazon and Rio Negro (1853).

3. Traduction de l'auteur de l'article. Le terme «Kikuyu » désigne à la fois le peuple Kikuyu, la langue, la ville de Kikuyu et la région.

4. Bruin est le surnom donné par les Pyrénéens à l'ours, qu'ils nommaient aussi Dominique ou Monsieur, ce qui montre le respect qu'ils avaient, à l'instar des Inuit, pour l'animal.

5. Je suis reconnaissante à Céline Roland Nabuco (qui cite ce passage de l'ouvrage d'Ortega, page 32) pour l'éclairage qu'elle apporte à la dimension profonde d'une certaine forme de chasse révélant l'homme à lui-même, dans sa belle thèse «Figures, gestes et cibles du sportsman. La chasse dans les textes de Jim Harrison et Thomas McGuane ».

6. <http://www.english.illinois.edu/maps/poets/m_r/momaday/buffalotrust.htm>, consulté le 8 juin 2016.

7. Les quatre critères selon Laurence Buell pour qu'un texte fasse partie de la littérature environnementale sont les suivants : - «Environmental writing, in contrast to nature writing, assumes the presence of natural history in human history."; - Environmental texts "open spaces for the nonhuman and its 'interests', sometimes privileging a non-androcentred world and its distinct evolution and history. "; - " Environmental writing imports into the text an ethical orientation that makes human beings responsible for the environment and accountable for its health and continuation.»; - "The environmental text assumes the processual order of nature and critiques or avoids a static model of natural change and ecological transformations. » (Buell, $1996: 7-8$ ).

8. Discours de Ken Saro-Wiwa, poète et militant nigérian, à propos de la lutte pour sa région dans le Delta du Niger pour le peuple Ogoni. Le texte de ce discours a été publié sous le titre «Ken Saro-Wiwa's final address to the military-appointed tribunal », Earth Island Journal (1995), 11(1), 25, Wikisource : <https://en.wikisource.org/wiki/Trial_Speech_of_Ken_Saro-Wiwa> (consulté le 18 mars 2016). 


\section{RÉSUMÉS}

Cet article vise à montrer le rôle du récit de voyage et d'ascension dans la conscience de la relation entre l'humain et le non humain et le passage de la relation synonyme de récit à la relation envisagée comme lien. Le récit de voyage ne s'enferme ni dans une aire géographique ni dans une période: qu'il s'agisse de voyageurs britanniques dans les Pyrénées, d'explorateurs anglais au cœur de l'Arctique ou d'un romancier amérindien racontant un voyage initiatique aux États-Unis, tous montrent dans leur récit la relation de l'homme au monde, car le genre même, fondé sur l'observation, en accordant autant d'importance à l'humain qu'au non humain, révèle le lien de l'homme au monde.

The article aims at showing the role of travel books in the awareness of the relationship between the human and non human through travel narratives. Travel writing is neither imprisoned in a geographical area nor in a temporal period. British travellers in the Pyrenees as well as English explorers in the Arctic, or a Native American novelist reporting an initiatory journey, all show the relationship of man and the world in their narratives, as the genre, founded on observation, reveals the link between man and the world.

\section{INDEX}

Keywords : Arctic, Canada, Native American literature, N. Scott Momaday, North West Passage, Pyrenees, mountain writing, travel writing, exploration writing, Rudy Wiebe

Mots-clés : Arctique, Canada, littérature amérindienne, N. Scott Momaday, passage du NordOuest, Pyrénées, récit d'ascension, récit de voyage, récit d'exploration, Rudy Wiebe

\section{AUTEUR}

\section{FRANÇOISE BESSON}

Professeur à l'Université Toulouse 2 - Jean Jaurès 